Journal of Engineering and Applied Sciences 14 (Special Issue 3): 6165-6174, 2019

ISSN: 1816-949X

(C) Medwell Journals, 2019

\title{
Errors and Misconceptions in Algebra: A Case Study of Pre-Commerce Students at UiTM Sarawak
}

\author{
Ting Su Ung, Ling Siew Eng and Chen Chee Khium \\ Faculty of Computer and Mathematical Sciences, Universiti Teknologi MARA, \\ UiTM Samarahan Campus, 94300 Samarahan, Sarawak, Malaysia \\ tsu_nic@sarawak.uitm.edu.my, lingse@sarawak.uitm.edu.my, ckchen@sarawak.uitm.edu.my
}

\begin{abstract}
Identifying errors in algebra provide information on misconceptions in algebra. This information is importation for educators to plan for the interventions program. The study identified the errors done by pre-commerce students in algebra. Explanatory design method was utilized and qualitative data were gathered from pre-commerce students. An algebraic assignment and two tests were used as instruments to identify errors and similar errors were coded under same theme. Students were interview to clarify the method used in obtaining their answers. The study identified five types of common errors in algebra by the students. The types of error were conjoin error, misapplication of rules, misinterpretation of cancellation, misuse of distributive property and sign error. The five types of error were related to the misconception on deficiency of prerequisite facts and concepts of algebra, could not systematically apply mathematical rules due to lack of basic understanding of algebraic expressions, incomplete understandings of arithmetical concepts, could not transfer arithmetic understanding to algebraic context and incompetence in dealing with integers and sign.
\end{abstract}

$\underline{\text { Key words: Algebra, error, misconception, Mathematics, algebraic assignment, incomplete understandings }}$

\section{INTRODUCTION}

Students make errors in solving Mathematics problems. The errors can be categorized into systematics, random and careless error. The systematic errors were due to misconceptions (Sarwadi and Shahril, 2014). The random errors arised when no relevant support on the student's thinking process and no mathematical rules imposed from the process of obtaining the solutions on the errors make (Cox, 1975). The careless errors occurred when students know how to perform the Mathematics solutions but make one or two mistakes in the process of obtaining the solution (Cox, 1975). From the 3 categories of errors, only systematic errors can provide further information on student's misconception. Therefore, it is important to identify student's systematic errors in solving Mathematics problem and further identify the misconceptions in Mathematics. Since, misconception is the main factors to student's performance (Aygor and Ozdag, 2012), therefore, intervention to correct student's misconception need to be carried out before they carry the wrong concepts to the higher levels of education (Kucuk, 2011; Titus, 2010).

Algebra is one of the important topics in Mathematics. Mulungye (2016) defined algebra as a branch in school Mathematics in which letters is used to represent quantities. In Malaysia, algebra is introduced to students from primary level to tertiary level. Since, algebra knowledge is widely used, therefore, algebra is a stepping stone for future career paths (Ralston et al., 2018; Lucariello et al., 2014). As the topic is abstract, students make many systematics errors as pointed out by a few researchers (Byrd et al., 2015; Bush and Karp, 2013; Cangelosi et al., 2013).

The intake of pre-commerce students at Universiti Teknologi MARA, Sarawak Branch (UiTMCS) is a program to give second chance education for students who do not fulfil the minimum entry requirement for tertiary study (Siew-Eng et al., 2015). The students are either weak in Mathematics or English. They were given one semester to improve their mathematical skills and English proficiency. Therefore, identify their errors make in Mathematics and further find out student's misconceptions may give lecturers information to plan and strategies their teaching method for this group of students.

This study aims to identify the systematic errors done in algebra by the pre-commerce students taking intensive Mathematics at Universiti Teknologi MARA, Sarawak Branch (UiTMCS). The study further identified misconceptions in algebra from the errors done by students.

Corresponding Author: Ting Su Ung, Faculty of Computer and Mathematical Sciences, Universiti Teknologi MARA, UiTM Samarahan Campus, 94300 Samarahan, Sarawak, Malaysia, tsu_nic@sarawak.uitm.edu.my 
Literature review: Studies on error and misconception started in decades ago to identify student's problem in Mathematics. Egodawatte (2011) defined error as a simple lapse of care done by students occasionally in solving Mathematics problem and misconception was defined by Bush (2011) as student's problem in understanding main concepts in algebra. Lucariello et al. (2014) explained the misconceptions in algebra included lack of knowledge in algebra, misunderstanding the concept and received inconsistent concepts in class.

Few researchers had embarked in error and misconception studies in algebra. Different researcher grouped the errors in a different ways. Lim (2010) reported 11 types of error in algebra as follows:

- Exponent error

- Negative sign error

- Misinterpretation of symbolic notation error

- Conjoin error

- Subtraction of integer error

- Addition of integer error

- Distributive error in bracket expansion

- Multiplication of variables error

- Like and unlike term error

- Negative pre-multiplier error

- Careless error

Bush (2011) categorized errors into five types as:

- The incorrect use of signs and omitting negative signs (numbers and numerical operations)

- Difficulty combining like terms (algebraic symbolism and letter usage)

- The difficulty with the symbolic representation of a scenario

- Understands the process of solving equations but makes a computational error

- Difficulty using inverse operations (algebraic equations) follows:

Booth et al. (2014) grouped errors into six types as

- Variable (like terms, unlike terms)

- Negative signs

- Equality (deleting or adding the equal sign)/inequality (the direction of inequality sign)

- Mathematical operations (illegal applying the addition, subtraction, multiplication or division)

- Mathematical property (inappropriately applying the commutative, associative or distributive property)

- Fraction (illegal simplification for the numerator and denominator)
Ncube (2016) identified his student's errors in algebra and categorized the errors into six types. There were conjoin error, misapplication of rules, misinterpretation of symbolic notation, invalid distribution of brackets, sign errors and substituting letters by numeric values. The six types of errors and the possible misconceptions are explained as follows:

Conjoin error: Conjoin error occur when one wishes to combine a term with another term through addition and subtraction. In algebra, to combined terms, students need to understand the concept of like and unlike term. For example, the terms $\mathrm{a}$ and $\mathrm{b}$ cannot be joined together in addition and subtraction. If students combined different terms by addition and substation, the students committed the conjoin error.

Misapplication of rules: When students do not apply the mathematical rules correctly, they committed misapplication of rules. For example, if $\frac{x}{y}+\frac{w}{z}$ is simplified to $x z+w y$, students misapplied the rule. ${ }^{y}$

Misinterpretation of symbolic notation: This type of error committed when students assumed an invisible coefficient occurs. For example, $2 a+a=2 a$. In this case, the student assumed the coefficient of a is 0 . Beside this, cancellation errors which are common among errors in algebra are also categorized under this category. For example, $\frac{x y}{x}+\frac{x z}{p x}$ is simplified to $(\mathrm{y}+\mathrm{z}) / \mathrm{p}$ by cancel all $\mathrm{x}$. The student who did the error reflected that he/she has some basic knowledge but could not organize it systematically.

Invalid distribution of brackets: This error often committed when expanding expression by applying distribution law. For example, $2(3 x-4 y)=6 x-4 y$. When expanding two expressions, some students ignore one of the terms and others might only expand one expression with one of the terms from the other expression. $(x-y)^{2}=x^{2}-$ $y+y^{2}$ or $(x-y)^{2}=x^{2}-x-y^{2}$ or $(x-y)^{2}=x^{2}-y^{2}$ are examples of invalid distribution of brackets.

Sign errors: Sign errors are one of the most common errors make by students. Ncube (2016) referred to this type of error as incorrect use of signs and omitting negative sign. The error was caused by a problem with operation sign and integers. To expand $-x(y-3)$, the student gets $-x y-3 x$. The error in the second term sign reflected the student misunderstood the sign.

Substituting letters by numeric values: When students substitute a letter by numeric values which they assumed they committed substituting letters by numeric values error. For example, if given $x+y=6$, students 
Table 1: Summary of the errors in algebra literature

Types

Conjoin error (Ncube, 2016)

Misapplication of rules (Ncube, 2016) Misinterpretation of symbolic notation (Ncube,

Sign errors (Ncube, 2016)
Other related name used in literature

Ignore the variable (Lucariello et al., 2014)

Like and unlike term error (Lim, 2010)

Difficulty combining like terms (algebraic symbolism and letter usage) (Bush, 2011)

Variable error of like term, unlike term (Booth et al., 2014)

Careless error (Lim, 2010)

Understand the process of solving equations but makes a computational error (Booth et al., 2014)

Incorrect order of operations (Lim, 2010)

The misapplication of BODMAS rule (Lim, 2010)

Subtraction of integer error (Lim, 2010)

Multiplication of variables error ( $\mathrm{Lim}, 2010)$

Mathematical operations (Illegal applying the addition, subtraction, multiplication or division) (Booth et al., 2014)

Misinterpretation of symbolic notation error (Lim, 2010)

Distributive error in bracket expansion (Lim, 2010) the difficulty with the symbolic representation of a scenario (Bush, 2011)

Mathematical property (Inappropriately applying the commutative, associative or distributive property) (Booth et al., 2014)

Negative sign errors (Lim, 2010)

Negative pre-multiplier error (Lim, 2010)

The incorrect use of signs and omitting negative signs (Numbers and numerical operations) (Bush, 2011)

Negative sign error (Booth et al., 2014)

Negative sign and indices that involving negative power (Cangelosi et al., 2013)

Difficulty using inverse operations (Bush, 2011)

Equality (deleting or adding the equal sign)/inequality (direction of inequality sign) (Booth et al., 2014)

Substituting letters by numeric values (Ncube, 2016)

answer $x+y+z=9$ because they assumed all letters were given equal to 3 . Overall, the types of errors in the above literature are summarized in Table 1.

Some types of errors classified by Ncube (2016) were similar to Lim (2010), Bush (2011) and Booth et al. (2014). In this study, the framework of Ncube (2016) was used to analysis the errors in algebra.

\section{MATERIALS AND METHODS}

Explanatory design method was utilized where both quantitative and qualitative data were gathered. The study is a case study where a group of 26 pre-commerce students registered intensive Mathematics course at Universiti Teknologi MARA, Sarawak Branch during semester June 2015-November 2015. An algebraic assignment and two sets of the test were used as the instruments to collect the data whilst the interview protocol was used to guide the interviewer to gather more information on the errors done. The test scripts were collected right after the students sat for the tests and the students were given a week to complete the assignment.

Student's test papers and their assignment were scrutinized to identify errors done by them. Ncube (2016) framework of six types of error was used to categorize the errors done by students. The six types of errors were conjoin error, misapplication of rules, misinterpretation of symbolic notation, invalid distribution of brackets, sign errors and substituting letters by numeric values.
Qualitative data were gathered through interview where the students were randomly selected for interview to establish the students thinking process in making the identified errors. The interview was carried out during the evening or weekends to avoid interruptions on student's daily schedule. In identified errors done by students, the analysis involved coding the errors into the six types of errors. The counting of the number of errors done in each category was also carried out.

\section{RESULTS AND DISCUSSION}

From the qualitative data, the study identified types of errors in algebra done by students. The five types of errors were conjoin error, misapplication of rules, misinterpretation of cancellation, misuse of distributive property and sign error. The qualitative data explained the thinking process of students on the errors done by them and the quantitative data summarized the descriptive statistics on each type of error found.

Conjoin error: There were $30.8 \%$ of the respondents exhibited the conjoin error. Below are the examples of errors done by the respondents. The following algebraic question was given to the respondents. Given the function $f(x)=2 x^{2}-6 x$, find $x$ if $f(x)=0$.

Figure 1 displays the solution of Respondent $R_{1}$. Figure 2 shows Respondent $\mathrm{R}_{2}$ solution. From the errors done by respondents as in Fig. 1 and 2, respondents 


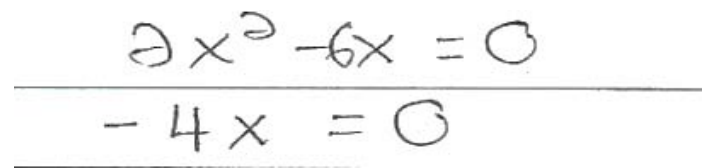

Fig. 1: Conjoin error done by Respondent $\mathrm{R}_{1}$

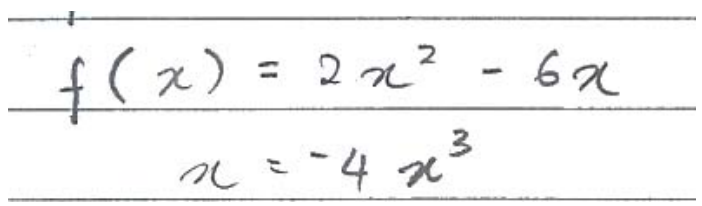

Fig. 2: Conjoin error done by Respondent $\mathrm{R}_{2}$

$$
\begin{aligned}
& =\frac{2 x+2}{x-2} \times \frac{2 x+1}{3 x-3} \\
& =\frac{4 x}{x-2} \times \frac{3 x}{x}
\end{aligned}
$$

Fig. 3: Conjoin error done by Respondent $\mathrm{R}_{3}$

faced problem to differentiate like and unlike terms. Below are parts of the interview data where I refer to interviewer and $\mathrm{R}$ refer to the respondent:

- I: May I know how do you obtain your answer $-4 \mathrm{x}$ ? (Referring to $2 \mathrm{x}^{2}-6 \mathrm{x}=-4 \mathrm{x}$ )

- $\mathrm{R}_{1}$ : I have subtracted 6 from 2 to get -4 . Next, I subtracted $\mathrm{x}$ from $\mathrm{x}^{2}$ to get $\mathrm{x}$. Then, I combined both parts of answer to get $-4 \mathrm{x}$

- I: Could you please tell me how you obtain your answer $-4 x^{3}$ ? (Referring to $2 x^{2}-6 x=-4 x^{3}$ )

- $\mathrm{R}_{2}$ : I have used calculator to get -4 by pressing 2-6. Next, I multiplied $\mathrm{x}^{2}$ by $\mathrm{x}$ to get $\mathrm{x}^{3}$. Lastly, I combined both answers together

Respondent $\mathrm{R}_{3}$ also exhibited conjoin error on the following question. Simplify:

$$
\frac{x^{2}-3 x+2}{x-2} \div \frac{3 x-2}{2 x+1}
$$

Figure 3 shows the solution produced by Respondent $R_{3}$. There were three conjoin errors found in Fig. 3. The first error was simplification done on $2 x+1=$ $3 \mathrm{x}$. Another two errors were $3 \mathrm{x}-3=\mathrm{x}$ and $2 \mathrm{x}+2=4 \mathrm{x}$. Below are parts of the transcription:

$$
\begin{aligned}
& -1<\frac{1-2 x}{3} \leqslant 2 \quad=-1<1 x \\
= & -1<\frac{1}{3} x \leq 2 \\
= & -1<\frac{1}{3} x \leqslant \frac{2}{3} .
\end{aligned}
$$

Fig. 4: Conjoin error done by Respondent $\mathrm{R}_{4}$

- I: May I know how do you obtain your answer 3x? (Referring to $2 \mathrm{x}+1=3 \mathrm{x}$ )

- $\mathrm{R}_{3}$ : I have added 2 with 1 to get 3 . Then, I copied the $x$. Therefore, I got my answer $3 x$.

- I: Do you use the same process to obtain $4 \mathrm{x}$ ? (Referring to $2 \mathrm{x}+2=4 \mathrm{x}$ )

- $\quad \mathrm{R}_{3}$ : Yes, I did. $2+2$ and I get 4. Then, I carried forward the $\mathrm{x}$ to get my answer $4 \mathrm{x}$

- I: How do you simplify the expression $3 x-3$ to get $x$ ?

- $\mathrm{R}_{3}$ : I have subtracted 3 from 3 to get 0 . Then I carried forward the variable $\mathrm{x}$

Another Respondent $\mathrm{R}_{4}$ make a similar error while solving the following inequalities:

$$
-1<\frac{1-2 \mathrm{x}}{3} \leq 2
$$

Figure 4 shows Respondent $\mathrm{R}_{4}$ 's solution. The following are parts of the interview transcription.

- I: Do you mind to share with me how do you obtain the value $1 \mathrm{x}$ ? (Referring to $1-2 \mathrm{x}=1 \mathrm{x}$ )

- $\mathrm{R}_{4}$ : First, $\mathrm{I}$ have subtracted 2 from 1 to get 1 . Then, I just copied the $\mathrm{x}$. Lastly, I combined both to get $1 \mathrm{x}$

The explanation given by the respondents indicated that they could not differentiate between like terms and unlike terms and assumed both terms were like terms. The errors done by Respondents $\mathrm{R}_{3}$ and $\mathrm{R}_{4}$ indicated that they were deficiency in algebra's concepts. They mixed up the variable $\mathrm{x}$ with multiply operation. Beside this, they interpreted the "-" sign as minus operation to solve. The respondents also confused between the algebra terms and the numeric terms. They only performed the addition and subtraction operations on numbers and ignored the algebra letter. They only added the algebraic letter after they had performed the operation as illustrated in Fig. 1-4. Ncube (2016) concluded the students who make this type of error have a deficiency of prerequisite facts and concepts of algebra.

Misapplication of rules: They were $53.8 \%$ of the respondents committed misapplication of rules. Figure 5 


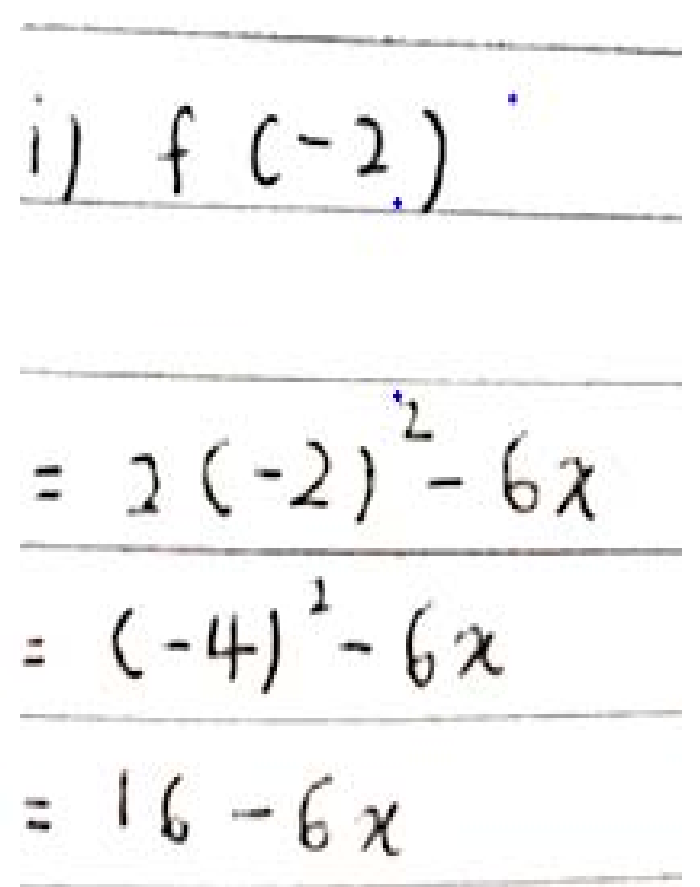

Fig. 5: Misapplication of rules done by Respondent $R_{5}$

shows the error on misapplication of rules in the following question. Given the function $f(x)=x^{2}-6 x$, find $f(-2)$. Figure 5 displays the solution by Respondent $\mathrm{R}_{5}$.

To better understand the respondent thinking, he was asked to clarify his solution. Below are extracted from the interview transcription:

- I: Could you please let me know how do you calculate $2(-2)^{2}$ to obtain $(-4)^{2}$ ?

- $\mathrm{R}_{5}$ : I just multiplied 2 by -2 in the bracket to get -4 . Then I copied the square

The respondent was confused among rules involving multiplication, brackets and powers. Another misapplication of rules was seen in the following solution by Respondent $\mathrm{R}_{6}$ (Fig. 6) in the following question. Given the function $f(x)=2 x^{2}-6 x$. Find $x$ if $f(x)=0$. The following extracted from the interview with Respondent $\mathrm{R}_{6}$ :

- I: Would you like to share with me how you solve this question?

- $\mathrm{R}_{6}$ : First, I have brought $2 \mathrm{x}$ to the right side of the equation and left the power of 2 on the right side of the equation. Then, I minus $2 \mathrm{x}$ from $6 \mathrm{x}$ to get $4 x$. After that, I calculated the square root of 4 to get 2 . Finally, I saw the $2 \mathrm{x}$ and I assumed that is $x=2$

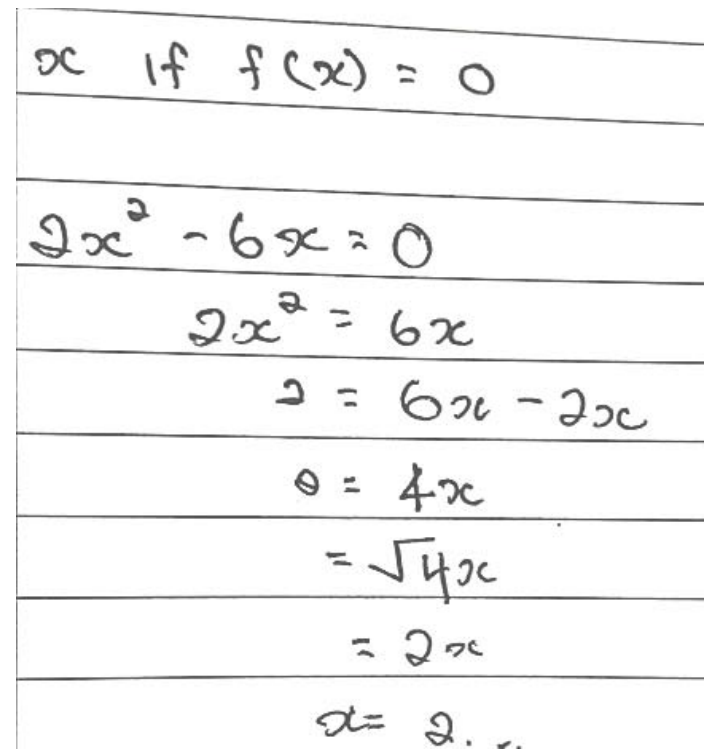

Fig. 6: Misapplication of rules done by the Respondent $\mathrm{R}_{6}$

$\begin{aligned} f(-2) & \\ f(-2) & =2(-2)^{2}-6(-2) \\ & =-4^{2}-12 \\ & =-16-12 \\ & =-28\end{aligned}$

Fig. 7: Misapplication of rules done by Respondent $R_{7}$

Respondents $\mathrm{R}_{7}$ produced the following solution Fig. 7 for the question. Given the function $\mathrm{f}(\mathrm{x})=2 \mathrm{x}^{2}-6 \mathrm{x}$. Find $f(-2)$. To better understand Respondent $R_{7}$ thinking process in producing the solution, a short interview was carried out. Below are extracted from the interview transcription:

- I: Would you like to tell me how you get this? (Referring to $2(-2)^{2}=-4^{2}$ )

- $\mathrm{R}_{7}$ : I have multiplied the number 2 with -2 in the bracket to get -4 . Then, I bought the power of 2 to the next step of the answer

- I: How do you calculate $-6(-2)$ to get -12 ?

- $\mathrm{R}_{7}$ : I have multiplied 6 by 2 to get 12 and $\mathrm{I}$ copied the negative sign and place it in front of number 12

The following inequalities question was given to Respondent $\mathrm{R}_{8}$ to solve. Figure 8 shows his solution. 


\begin{aligned} $2(x-3)<-1 \\$\hline$(x-3)<-1-2 \\$\hline$x-3-<-3 \\$\hline$x>-3 \\$\hline$x>1\end{aligned}$

Fig. 8: Misapplication of rules done by Respondent $\mathrm{R}_{8}$

Below is the transcriptions captured during the interview session with $\mathrm{R}_{8}$. He explained how he obtained his solution:

- I: Could you explain in details what you actually do to the number 2 in front of the bracket?

- $\mathrm{R}_{8}$ : I saw the 2 in front (left-hand side) is a positive number 2 , so, I deleted the number 2 by subtracting 2 from both sides of the inequality

- I: Ok. Now, please let me know how do you solve the -3 in the fourth line of your answer?

- $\mathrm{R}_{8}$ : I wanted to eliminate it. So, I just divided the -3 on the left side of the inequality with -3 and performed the same process to the -3 on the right side of the inequality

Respondent $\mathrm{R}_{8}$ was confused about the rules of Mathematics. He knew that he have to perform the operation for number 2. Unfortunately, he performed the subtraction instead of multiplication. He made the same mistake on the following step where he performed the multiplication instead of subtraction. This indicated that Respondent $\mathrm{R}_{8}$ was confused about the rules of operation.

The error done by Respondent $R_{8}$ was on the misapplication of rules. From the errors done by respondents as in Fig. 5-8, the respondents have some knowledge of the rules but misapplied the rules in solving Mathematics problem.

Misinterpretation of cancellation: There were 34.6\% of the students contributed to misinterpretation of cancellation error. Most of the students understand that they need to simplify the fractions but they fail to simplify. The following were errors made by the respondents in the following question. Simplify:

$$
\frac{x^{2}-3 x+2}{x-2} \div \frac{3 x-2}{2 x+1}
$$

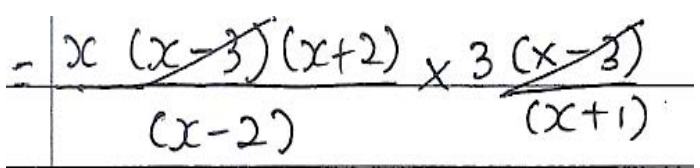

Fig. 9: Misinterpretation of cancellation done by Respondent $\mathrm{R}_{9}$

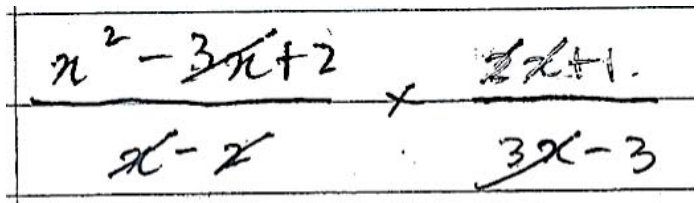

Fig. 10: Misinterpretation of cancellation by Respondent $\mathrm{R}_{10}$

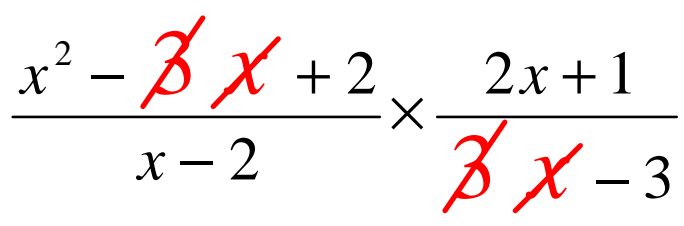

Fig. 11: Misinterpretation of cancellation by Respondent $\mathrm{R}_{10}$

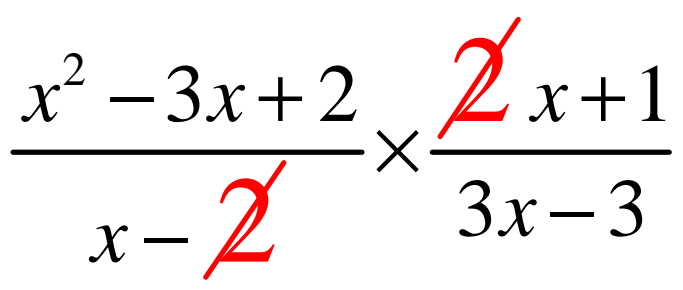

Fig. 12: Misinterpretation of cancellation of number ' 2 ' done by $\mathrm{R}_{10}$

Figure 9 and 10 show the answers given by Respondent $R_{9}$ and $R_{10}$, respectively. Below are parts of the conversation between Respondent $R_{9}$ and interviewer on the error showed in Fig. 9:

- I: May I know why you eliminate the term ' $x-3$ ' on the both sides of the fraction?

- $\mathrm{R}_{9}$ : Oh, this is because it is the same terms. I only know I cannot eliminate the same terms or expressions within the same fraction but I can eliminate it between different fractions regardless the term is a numerator or a denominator

Figure 10 illustrated Respondent $\mathrm{R}_{10}$ 's solution. Figure 10 Respondent $\mathrm{R}_{10}$ did three cancellations in his solutions in simplifying the fractions. The errors done in the cancellation were illustrated from Fig. 11-13 as follows. 


\section{$\frac{x^{2}-3 x+2}{x-2} \times \frac{2 \not x+1}{3 x-3}$}

Fig. 13: Misinterpretation of cancellation of ' $\mathrm{x}$ ' term done by Respondent $\mathrm{R}_{10}$

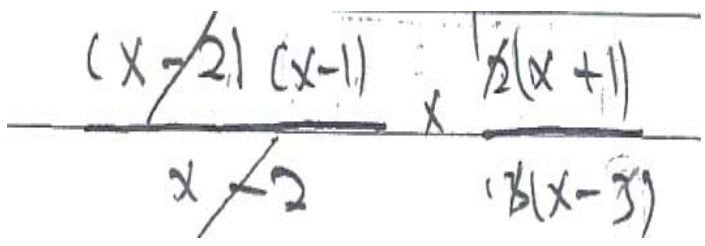

Fig. 14: Misinterpretation of cancellation done by Respondent $\mathrm{R}_{11}$

The evidence can be justified accordingly to the transcription recorded, respectively. First, he eliminated the term 3x (Fig. 11). Next, he eliminated the coefficient of 2 (Fig. 12) and lastly, he cancelled the $x$ term (Fig. 13). Below are the explanations of Respondent $R_{10}$ on his cancellation on term $3 \mathrm{x}$, term 2 and term $\mathrm{x}$ :

- I: Could you please explain to me how do you eliminate ' $3 \mathrm{x}$ '?

- $\mathrm{R}_{10}$ : I have cancelled it because it was the same terms I found in the fractions

- I: How about the number ' 2 '?

- $\mathrm{R}_{10}$ : I have cancelled it too. There is '2' on the left fraction and ' 2 ' on the right fraction. (Pointed at the $2 \mathrm{~s}$ cancelled). They are the same terms

- I: How about the ' $\mathrm{x}$ ' term?

- $\mathrm{R}_{10}$ : I have eliminated it, since, there are same $\mathrm{x}$ terms, one on the left fraction and one on the right fraction

Another Respondent $\mathrm{R}_{11}$ performed a similar type of error as shown in Fig. 14. For better understand the respondent's thought, the interview has been carried out and below are parts of the conversation between the interviewer and Respondent $\mathrm{R}_{11}$ :

- I: Could you explain to me why you cancel both of the number 3 ? (Pointing to the expression 3(x-3) on the denominator at the second fraction)

- $\quad \mathrm{R}_{11}$ : Yes, I have cancelled both number 3 and left $\mathrm{x}$. (Demonstrated her steps of the solution on a piece of paper)

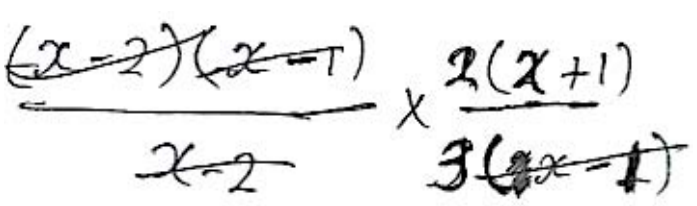

Fig. 15: Misuse of the distributive law (Factorization) done by Respondent $\mathrm{R}_{12}$

From the respondent's solution, they were identified as misinterpretation of the fraction. In simplifying, the fraction, they eliminated the same terms on numerator or denominator. They just eliminated any like terms they saw without considering either it is a term in an expression or a factor. They solve the question without considering the basic rules of the fraction. This indicated that the respondents lacked of basic understanding of algebraic expressions as mentioned by Ncube (2016).

Misuse of the distributive property: They were $46.2 \%$ of the respondents misuse of the distributive property law. Figure 15 shows Respondent $\mathrm{R}_{12}$ 's solution when simplifying the following question. Simplify:

$$
\frac{x^{2}-3 x+2}{x-2} \div \frac{3 x-2}{2 x+1}
$$

The student factorized two expressions which cannot be factorized. They factorized $2 x+1$ as $2(x+1)$ and $3 x-2$ as $3(\mathrm{x}-2)$. To understand the student thinking process, we ask him to explain his answer. Below are the conversations extracted from the interview:

- I: Could you please explain to me how do you get $2 \mathrm{x}+1=2(\mathrm{x}+1) ?$

- $\mathrm{R}_{12}:$ I have factorized 2

- I: Alright. How do you get the number 1 in the bracket?

- $\mathrm{R}_{12}$ : I have maintained the number ' 1 ' inside the bracket

- I: Now, let me understand how you get $3 x-2=3(x-2)$

- $\mathrm{R}_{12}: \mathrm{I}$ did the same thing. I factorized 3 and $\mathrm{I}$ maintained the -2

Another Respondent, $\mathrm{R}_{13}$ 's solution for the same question is illustrated in Fig. 16. The error done by Respondent $R_{13}$ was $3 x-3=3(x-3)$. Below are parts of the interview transcription:

- I: May I know how you get $3 x-3=3(x-3)$ ?

- $\mathrm{R}_{13}$ : I have factorized the value 3

- I: How about this value 3 in the bracket? (Pointing the value 3 in the bracket)

- $\mathrm{R}_{13}$ : I just copied the value 3 


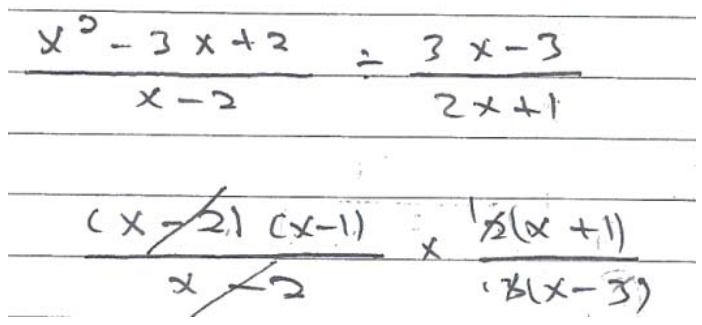

Fig. 16: Respondent $R_{13}$ 's error on the misuse of the distributive law (Factorization)

$$
\begin{aligned}
& (2 x-1)(2 x-1) \\
& (4 x-2 x-2 x+1)
\end{aligned}
$$

Fig. 17: Respondent $R_{14}$ 's error on the misuse of the distributive law (Expansion)

a) $\begin{aligned} & \text { simplify }(2 x-1)^{2}-2\left(x^{2}-2 x+1\right)-x(x+1) \\ = & (2 x-1)(2 x-1)-2\left(x^{2}-2 x+1\right)-x(x+1) \\ = & 4 x-2-2 x-2-2\left(x^{2}-1\right)-x^{2}-2 \\ = & 4 x-2-2 x-2-2 x^{2}+2-y^{2}-2 \\ = & 2 x-3 x^{2}-4 x\end{aligned}$

Fig. 18: Respondent $\mathrm{R}_{15}$ 's error on the misuse of the distributive law (Expansion)

The following question was given to Respondents $\mathrm{R}_{14}$ and $\mathrm{R}_{15}$. Simplify $(2 \mathrm{x}-1)^{2}-2\left(\mathrm{x}^{2}-2 \mathrm{x}+1\right)-\mathrm{x}(\mathrm{x}+1)$. Respondent $\mathrm{R}_{14}$ solution is shown in Fig. 17. The error done by Respondent $R_{14}$ was $(2 x)(2 x)=4 x$. Transcription below recorded the explanation on how Respondent $\mathrm{R}_{14}$ obtained her solution:

- I: Could you please explain to me how do you obtain the term $4 \mathrm{x}$ ?

- $\mathrm{R}_{14}$ : I have multiplied 2 by 2 to get 4 . Then, I copied the $\mathrm{x}$ to get $4 \mathrm{x}$. I felt that $\mathrm{x}$ is the same in the two terms, so, I just copied it there

Figure 18 shows the solution of the Respondent $\mathrm{R}_{15}$. The error committed by Respondent $\mathrm{R}_{15}$ was (2x-1) $(2 x-1)=4 x-2-2 x-2$. Transcription below recorded Respondent $R_{15}$ 's thinking process on his solution:

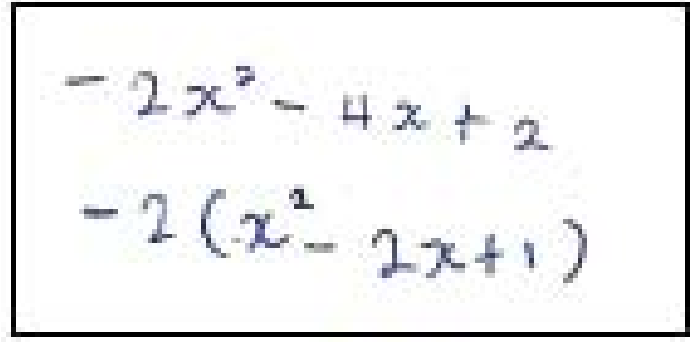

Fig. 19: Sign error done by Respondent $\mathrm{R}_{16}$

- I: Do you mind to share with me how do you actually obtain your answer?

- $\mathrm{R}_{15}$ : Sure, no problem, madam

- I: Could you please explain to me how do you to get the first term $4 x$ ?

- $\mathrm{R}_{15}$ : I have multiplied 2 by 2 to get 4 and maintained the $x$

- I: Please share with me how do you obtain the second term -2 ?

- $\mathrm{R}_{15}$ : I just multiplied 2 by -1 to get -2

- I: How about the existing $\mathrm{x}$ term?

- $\mathrm{R}_{15}$ : I just ignored it because I was confused

- I: Could you please explain to me how do you obtain the fourth term -2 ?

- $\mathrm{R}_{15}$ : I have multiplied -1 by -1 to get -2 . I am not sure too, madam

This type of error revealed that the respondent misuse of the distributive law which cause by lack of comprehensive understanding of algebraic ideas (Ncube, 2016; Mulungye, 2016) or incomplete understandings of arithmetical concepts and failure to transfer arithmetic understanding to algebraic context (Mulungye, 2016).

Sign error: Students make a sign error when it involved terms with a negative coefficient. This type of errors found in $38.5 \%$ of the pre-commerce students. For example, in simplify $(2 \mathrm{x}-1)^{2}-2\left(\mathrm{x}^{2}-2 \mathrm{x}+1\right)-\mathrm{x}(\mathrm{x}+1)$, Respondents $R_{16}$ and $R_{17}$ and $R_{18}$ done the following errors as shown in Fig. 19-21, respectively.

This study found that the respondents make this type of error when they try to open the bracket by applying the distribution law. Respondents $\mathrm{R}_{16}$ explained how he obtained his solution as follows:

- I: May I know how do you get this answer: $-2\left(x^{2}-2 x+1\right)=-2 x^{2}-2 x+2$

- $\mathrm{R}_{16}$ : I have multiplied every term in the bracket by 2

- I: If you multiply each term by 2 , how do you get second term $-2 \mathrm{x}$ 


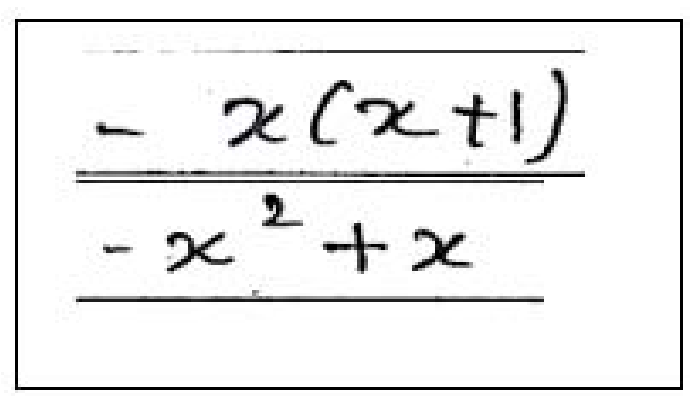

Fig. 20: Sign error done by Respondent $R_{17}$

(i) \begin{aligned}$f(-2) & \\$\hline$f(-2=x) & =2 x^{2}-6 x \\$\hline$f(-2) & =2(-2)^{2}-6(-2) \\ & =8-12 \\ & =-4\end{aligned}$

Fig. 21: Sign error done by Respondent $\mathrm{R}_{18}$

- $\mathrm{R}_{16}$ : For this term, $\mathrm{I}$ saw the coefficient 2 is there already, so, I just copied this term only

- I: Could you please explain to me what do you do with the negative sign in the -2 term in front of the bracket?

- $\mathrm{R}_{16}$ : I just copied the negative sign to the first term of the answer

The following are the conversation extracted from Respondent $\mathrm{R}_{17}$ explanation:

- I: Could you please explain to me how do you expand $-\mathrm{x}(\mathrm{x}+1)$ ?

- $\mathrm{R}_{17}$ : I have expanded the expression by multiplying each term in the bracket by $x$

- I: Do you notice that there is a negative sign in front of the $\mathrm{x}$ term?

- $\mathrm{R}_{17}$ : Yes, I did

- I: What you do with this negative sign in - $\mathrm{x}$ term?

- $\mathrm{R}_{17}$ : I just copied it in the first term of my answer to get $-\mathrm{x}$

Beside this, another Respondent $\mathrm{R}_{18}$ has done the same type of error on the following question. Given the function $f(x)=2 x^{2}-6 x$. Find $f(-2)$. His answer is shown in Fig. 21. Below is part of the explanation on his thinking process:
- I: Could you please explain in details how do you obtain your answer -12 ?

- $\mathrm{R}_{18}$ : First, $\mathrm{I}$ have used the calculator and press 6 multiply by -2 . This is how I get my answer. (Demonstrate by pressing the answer on the calculator)

- I: How about the negative sign in front of the number 6 ?

- $\mathrm{R}_{18}:$ I just ignored it

Students were seen depend on calculator in most of their solution processes including performing simple operations. They used the calculator in solving Mathematics problems but did not relate it to the mathematical concepts and rules. The finding was supported by Ruthven and Chaplin (1997). They revealed that calculator was a helpful tool for students in the calculation process but students need know the correct concepts in Mathematics before they apply it.

The study found that the respondents have problems with direction signs as shown in Fig. 19-21. This indicated that the student's confused with signs which caused them to be incompetence in dealing with integers (Ncube, 2016).

\section{CONCLUSION}

There were many errors done by students in Mathematics problems solving. In this study, five types of errors in algebra done by pre-commerce students were identified. The five types of errors were conjoined error, misapplication of rules, misinterpretation of cancellation, misuse of distributive property and sign error.

The collection of qualitative data through interview was to understand student's thinking process of solving the given problems. From there, the misconception of students can be identified and intervention can be arranged to tacker those concepts which were perceived wrongly by students. The errors done by students were due to several misconceptions such as deficiency of prerequisite facts and concepts of algebra could not systematically apply mathematical rules due to lack of basic understanding of algebraic expressions, incomplete understandings of arithmetical concepts could not transfer arithmetic understanding to algebraic context and incompetence in dealing with integers and sign. The diagnosed misconceptions in algebra can play a vital role for the educators or lecturers to plan for the suitable intervention programmes tailored to this group of students to assist them to overcome the misconceptions. A similar study can be carried out on other Mathematics topics to understand the overall weaknesses of pre-commence students in Mathematics. 


\section{REFERENCES}

Aygor, N. and H. Ozdag, 2012. Misconceptions in linear algebra: The case of undergraduate students. Procedia Soc. Behav. Sci., 46: 2989-2994.

Booth, J.L., C. Barbieri, F. Eyer and E.J. Pare-Blagoev, 2014. Persistent and pernicious errors in algebraic problem solving. J. Prob. Solving, 7: 1-14.

Bush, S.B. and K.S. Karp, 2013. Prerequisite algebra skills and associated misconceptions of middle grade students: A review. J. Math. Behav., 32: 613-632.

Bush, S.B., 2011. Analyzing common algebra-related misconceptions and errors of middle school students. Ph.D Thesis, University of Louisville, Louisville, Kentucky.

Byrd, C.E., N.M. McNeil, D.L. Chesney and P.G. Matthews, 2015. A specific misconception of the equal sign acts as a barrier to childrens learning of early algebra. Learn. Individual Differences, 38: 61-67.

Cangelosi, R., S. Madrid, S. Cooper, J. Olson and B. Hartter, 2013. The negative sign and exponential expressions: Unveiling students persistent errors and misconceptions. The J. Math. Behav., 32: 69-82.

Cox, L.S., 1975. Systematic errors in the four vertical algorithms in normal and handicapped populations. J. Res. Math. Educ., 6: 202-220.

Egodawatte, G., 2011. Secondary school students misconceptions in algebra. Ph.D. Thesis, University of Toronto, Canada.

Kucuk, B., 2011. Identifying the secondary school students misconceptions about functions. Procedia Soc. Behav. Sci., 15: 3837-3842.
Lim, K.S., 2010. An error analysis of form 2 (Grade 7) students in simplifying algebraic expressions: A descriptive study. Electron. J. Res. Educ. Psychol., 8: 139-162.

Lucariello, J., M.T. Tine and C.M. Ganley, 2014. A formative assessment of students algebraic variable misconceptions. J. Math. Behav., 33: 30-41.

Mulungye, M.M., 2016. Sources of students errors and misconceptions in algebra and influence of classroom practice remediation in secondary schools machakos sub-county, Kenya. Master Thesis, Kenyatta University, Kahawa, North East.

Ncube, M., 2016. Analysis of errors made by learners in simplifying algebraic expressions at grade 9 level. Ph.D Thesis, University of South Africa, Pretoria, South Africa.

Ralston, N.C., M. Li and C. Taylor, 2018. The development and initial validation of an assessment of algebraic thinking for students in the elementary grades. Educ. Assess., 23: 211-227.

Ruthven, K. and D. Chaplin, 1997. The calculator as a cognitive tool: Upper-primary pupils tackling a realisticnumber problem. Intl. J. Comput. Math. Learn., 2: 93-124.

Sarwadi, H.R.H. and M. Shahrill, 2014. Understanding students mathematical errors and misconceptions: The case of year 11 repeating students. Math. Educ. Trends Res., 2014: 1-10.

Siew-Eng, L., R. Mahdi, M.I. Mohamadin and B.H.A. Manaf, 2015. Second chance science education for school leavers. Procedia Soc. Behav. Sci., 167: 288-292.

Titus, F., 2010. A cognitive analysis of developmental mathematics students errors and misconceptions in real number computations and evaluating algebraic expressions. Ph.D Thesis, University of Houston, Houston, Texas, USA. 